\title{
A TRIBUTE TO THE CONTRIBUTIONS OF PROFESSOR SHIGERU OAE IN HETEROCYCLIC CHEMISTRY
}

\author{
Waichiro Tagaki \\ Professor Emeritus Osaka City University \\ Sennichi-cho, Yamatokoriyama-shi, Nara 639-11, Japan
}

It is my great pleasure and an honor as one of junior associates of Professor Shigeru Oae to contribute the preface to this special issue which is dedicated to Professor Oae on the occasion of his 77th birthday, so called Kiju in Japan. It is really astonishing to us all that he has been continuing up to date as a forefront researcher and leader in the fields of HETEROATOM CHEMISTRY and HETEROCYCLIC CHEMISTRY as well, even after his retirement in 1983 at the age of 63 . Indeed he has published total 737 publications including 526 original papers, 32 books and 179 review articles, of which 76 original papers have been published after his retirement.

Professor William E. McEwen stated in his "A Tribute to Professor Shigeru Oae (Heteroatom Chemistry, Vol. 6, No. 1, 1995)" that as for Professor Oae retirement was such as to afford a maximum advantage of the new degrees of freedom. Indeed he soon resumed his scientific activity by means of an arrangement with the Okayama University of Science, by establishing the Institute of Heteroatom Chemistry in Osaka, by becoming the founding editor of REVIEWS ON HETEROATOM CHEMISTRY, by becoming the Far East Editor of HETEROATOM CHEMISTRY, by carrying out cooperative research work with many Japanese chemists, and by visiting many laboratories throughout the world to give lectures on "Ligand Coupling Reactions within Hypervalent Species".

Professor Oae has made many pioneering contributions in the field of physical organic chemistry. In particular, his achievements in the field of organic sulfur chemistry will be remembered long by future generations. The study on sulfur chemistry has naturally extended to include a more broad fjeld of heteroatom chemistry which of course overlaps with heterocyclic chemistry. On the following, the contributions of Professor Oae are briefly summarized. 


\section{(1) Acid-Enhancing Effect of Dicoordinate Sulfur Groups}

A series of studies of the acid-enhancing effect of $\alpha$-alkylthia or $\alpha$-arenethia groups on adjacent methylene group protons were reported for the first time in 1960 on the basis of quantitative kinetic evidence. The effect was shown to be multiplied in a logarithmic manner by further substitution of a second or third alkylthia or arylthia group at the $\alpha$-carbon in accord with the concept of electron-accepting $3 d$-orbital resonance effects. The bridgehead hydrogen of a bicyclic trisulfide was shown to be highly acidic as compared to that of a corresponding oxygen analog. This carbon acid-enhancing effect of an $\alpha$-thia group led to applications by many chemists, e.g., in the synthetic uses of 1,3-dithiane-2-lithio derivatives and to the rationalization of the markedly high catalytic reactivity of Co-enzyme A. Similarly, the $\alpha$-thia group was shown to stabilize free radicals, apparently by an electron-sharing conjugative resonance effect involving $3 \mathrm{~d}$-orbital of sulfur.

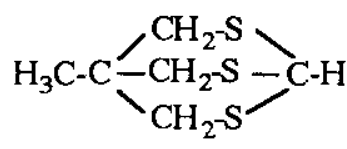

A bicyclic trisulfide<smiles>[R]C1([Tl])SCCCS1</smiles>

1,3-dithiane-2-lithio derivatives

\section{(2) Chemistry of Chiral Tricoordinate Organic Sulfur Compounds}

In 1967, Professor Oae initiated a research program to study the nature of tricoordinate organic sulfur compounds of general formula

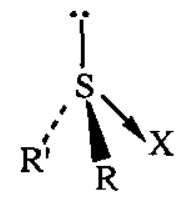

where $\mathrm{X}=\mathrm{O}, \mathrm{N}$ or $\mathrm{C}$

The chiral nature of the compounds was extensively exploited in mechanistic studies of isotopic oxygen exchange of sulfoxides, in substitution and rearrangement reactions of sulfoxides and sulfilimines, and thermal racemization of these and related compounds. Convenient methods to incorporate 180 into sulfoxides were utilized to open the way to study the stereochemistry of nucleophilic substitution at tricoordinate sulfur in sulfoxides. Sulfoxides were found to undergo oxygen exchange in sulfuric, phosphoric, and carboxylic acids, in $\mathrm{N}_{2} \mathrm{O}_{4}$, in carboxylic acid anhydrides, with alkali metal alkoxides, and 
with sulfoxides themselves. Especially interesting was the demonstration that optically active methyl phenyl sulfoxide underwent the oxygen exchange reaction with retention of configuration. This was the first demonstration of an $S_{\mathrm{N}} 2$ reaction on tricoordinate sulfur occurring with retention of configuration.

As an extension of the work with tricoordinate sulfur compounds, the chemistry of several seleno analogs has been explored. Among other findings, the first example of an optically active selenonium ylide was prepared.

\section{(3) Ligand Coupling within a Hypervalent Intermediate}

In 1984, S. Oae, T. Kawai, and N. Furukawa reported that the reaction of optically active $\alpha$-phenylethyl $\alpha$-pyridyl sulfoxide occurred with an alkyl or aryl Grignard reagent to give, in quantitative yield, an optically active coupling product 2-(phenylethyl)-pyridine with $100 \%$ retention of the configuration of the chiral carbon atom. This has led, extending up to the present time, to numerous additional studies of ligand

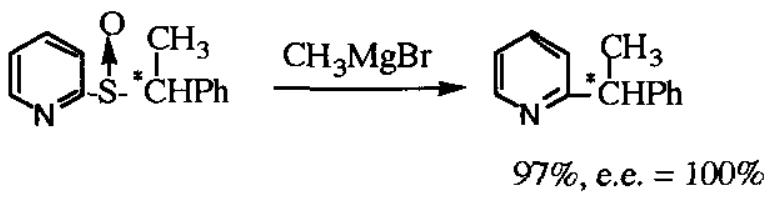

coupling reactions within hypervalent intermediates, and the basic concept of which has been summarized by Professor Oae in 1991 (S. Oae and Y. Uchida, Acc. Chem. Res., 1991, 24, 202-208). If the central atom of the reacting molecule is below the second row of the periodic table and can expand the valenceshell beyond an octet to form a hypervalent species, there is a possibility for ligand coupling to occur within this species. Thus it is probable that such reactions have been observed with compounds of more than two dozen different hetero atoms. In all of these cases, attack of a nucleophililic reagent at the central hetero atom occurs to give a hypervalent intermediate that subsequently undergoes concerted intramolecular coupling of an electron-withdrawing ligand located at an apical position with an electron-donating or $\pi$ ligand located at an equatorial position, these ligands having attained the necessary geometrical sites by prior pseudorotation, as reqired. In nearly all cases, retention of configuration of coupling ligands has been observed, and the stereochemical nature of the reaction can be used to diagnose the occurrence of ligand coupling, whenever there are some stereochemical data available. Since ligand coupling is stereospecific in most cases, the reaction can serve to supply stereochemically well defined compounds, and therefore it should be quite useful for synthetic applications. 


\section{(4) The Pummerer Reaction}

Treatment of a sulfoxide bearing an $\alpha$-hydrogen with acetic anhydride produces an $\alpha$-acetoxy sulfide, which in turn, can readily be hydrolyzed to an aldehyde plus a thiol. The mechanism of the rearrangement step was studied by Professor Oae and his coworkers in the early 1980 s by the use of D and $18 \mathrm{O}$ tracers and optically active or geometrically isomeric sulfoxides. The following four-step mechanism was proposed for the rearrangement:

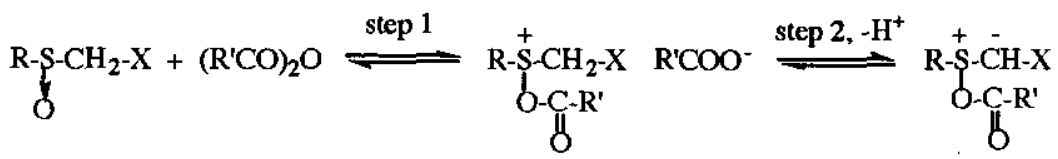

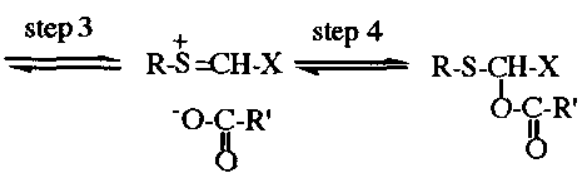

It was demonstrated that the 1,2-acyloxy shift proceeds in an intramolecular manner, with formation of an intimate ion pair, with sulfoxides in which $\mathrm{X}$ represents an elctron-withdrawing substituent, whereas the rearrangement proceeds primarily in an intermolecular manner when $\mathrm{X}$ is a simple alkyl group.

\section{(5) Sulfilimines and Sulfoximines}

Sulfilimines are the nitrogen analogs of sulfoxides. Professor Oae and his coworkers developed a convenient synthetic procedure for the conversion of sulfide to an $N$-tosylsulfilimine. Such sulfilimines, by analogy to the behavior of sulfoxides, were found to undergo stereospecific cis-elimination by simple pyrolysis. Facile Pummerer-type rearrangements, reductions, and halide-catalyzed rearrangements were similarly discovered. A procedure to prepare "free sulfilimines" was developed next, and this led to the development of a new field of sulfilimine chemistry. Their acylation and subsequent cleavage reactions gave isocyanates. Their addition to $\alpha, \beta$-unsaturated ketones gave $\alpha$-carbonylaziridines. Their oxidation gave sulfoximines that previously could be prepared only by a hazardous procedure. Their halogenation afforded $N$-halosulfilimines that undergo reaction with soft nucleophiles at nitrogen, but with hard nucleophiles at sulfur to give sulfoximines or sulfonediimines.

Optically active free sulfilimines were prepared for the first time, and these were found to be useful in establishing stereochemical reaction cycles. A facile synthesis of an optically active free diarylsulfilimine was achieved, and it could be utilized to prepare an optically active aziridine and an optically active 
sulfoximine. Also, a free diarylsulfilimine was found to react with an aldehyde to form a nitrile in quantitative yield.

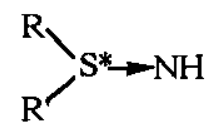

Optically active free sulfilimines

\section{(6) Chemical and Enzymatic Oxidation and Reduction of Organosulfur Compounds}

After some years of studies on the syntheses of sulfoxides from sulfides, and sulfonic anhydrides from disulfides and $\mathrm{N}_{2} \mathrm{O}_{4}$, Professor Oae began to investigate modes of oxidation of divalent sulfur compounds. In the oxidation of thiols, disulfides, and thiolsulfinates with $\mathrm{N}_{2} \mathrm{O}_{4}$, intermediate thionitriles $(-\mathrm{S}-\mathrm{N}=\mathrm{O})$ were isolated and found to be excellent diazotization agents. In the electrophilic oxidation of a thiolsulfinate with a peracid, evidence for the formation of an " $\alpha$-disulfoxide" intermediate was shown by use of ${ }^{18} \mathrm{O}$-labeled methylthiolbenzenesulfinate.

Enzymatic oxidation of sulfides was carried out successfully with purified cytochrome P-450. This oxidation involves an initial one-electron transfer from sulfur, similar to that with the Udenfriend system (ferrous ascorbate mixture).

Sodium metaperiodate was shown to oxidize unsymmetrical thiolsulfinates selectively to the corresponding thiolsulfonates, suggesting the metal oxides to be nucleophilic oxidizing agents. The modes of base-catalyzed air oxidation of thiols, disulfides, and thiolsulfinates were shown to involve both peroxysulfinates and peroxysulfenates and were similar to oxidations with the superoxide anion. Also, nucleophilic attack of hydroxide ion on thiolsulfinates was shown by the use of an $18 \mathrm{O}$ tracer to take place on the sulfinyl sulfur in the oxidative S-S bond cleavage.

On the basis of these and similar studies with various sulfur compounds, Professor Oae suggested that there are three different modes of oxidation of such compounds, viz., electrophilic, nucleophilic, and those involving SET (single-electron transfer), depending on the oxidants and media. The action of the cytochrome P-450 system, or of Fenton's reagent on a sulfide, with S-oxidation or dealkylation, was thought to involve the initial formation of a sulfenium cation-radical (A) by the SET mechanism. 


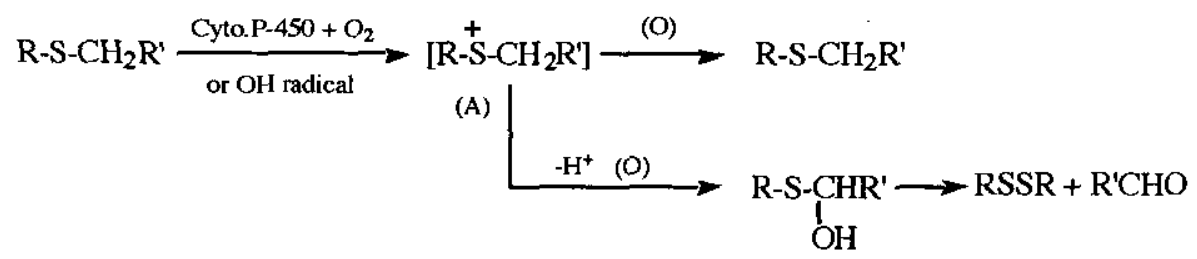

Peracids, singlet oxygen, $\mathrm{N}_{2} \mathrm{O}_{4}$, and FAD-monooxygenase are electrophilic oxidants, and $\mathrm{KIO}_{4}$, $\mathrm{KMnO}_{4}$, peracylates, and the superoxide anion are typical nucleophilic oxidants. The assumption that peroxysulfonates -sulfinates and -sulfenates are formed in the alkaline autoxidation of thiols and disulfides, and in the reaction of compounds containing the S-S linkage with the superoxide anion, was confirmed by trapping experiments.

Successful reductions of sulfoxides and sulfoximines with either elemental sulfur or disulfide, with HI and others have been reported. After many years of studies on the reduction of sulfoxides and related compounds, one-pot reduction of sulfonic and sulfuric acids was finally achieved by three different systems. Among these, polyphosphoric derivatives/KI or thiols system was found to be not only an effective but also interesting reductant which can reduce both sulfonic and sulfuric acids directly to thiols and hydrogen sulfide. Furthermore, formation of $\mathrm{SO}_{3}$ and $\mathrm{SO}_{2}$ as intermediates was confirmed by trapping experiments, thus clarifying the mechanistic pathway of the enzymatic reduction of sulfuric acid.

\section{(7) Rearrangements of Tertiary Amine Oxides and other Miscellaneous Reactions}

Both alkyl-substituted and unsubstituted heteroaromatic $N$-oxides react with various acylating agents and undergo interesting rearrangements. The mode of the rearrangement differs widely depending upon the $N$ oxides and the acylating agents. In 1960 Professor Oae initiated a research program to clarify the mechanisms of these rearrangements of various types using $18 \mathrm{O}$ tracers and H-D kinetic isotope effects. After 15 years the mechanisms of most of these rearrangements have been elucidated and several new types of rearrangements were discovered.

Professor Oae and his colleagues have also carried out extensive studies on reactions of elemental sulfur, mechanisms of reactions of phenols and phenolic esters, decomposition of diacyl peroxides, anchimeric assistance in nucleophilic substitution reactions of organic halides, and radiation and photochemistry of organosulfur compounds. Details of these studies can be found in the open literature and also in a private printing of a book by Professor Oae in 1983, entitled, Seeking Beyond the 
Horizon of Science. This book was published by a group of Professor Oae's former students under the management of Naomichi Furukawa.

\section{ACKNOWLEDGMENTS.}

Much of the material for this preface was dated from the above mentioned book of Professor Oae: Seeking Beyond the Horizon of Science and the article of Professor William E. McEwen: A Tribute to Professor Shigeru Oae. The author sincerely thanks Professors Naomichi Furukawa of Tsukuba University and Atsuyoshi Ohno of Kyoto University for their kind suggestions in preparing this preface. 